\title{
Temporal patterns of commonly used clinical outcome scales during a 5-year period after total knee arthroplasty
}

Vivek Tiwari ${ }^{1}$, Jonggeun Lee ${ }^{1}$, Gaurav Sharma', Yeon Gwi Kang ${ }^{1}$ and Tae Kyun Kim ${ }^{1,2^{*}}$

\begin{abstract}
Background: It is not established beyond doubt whether improvements in functional outcome after total knee arthroplasty (TKA) are maintained in the long term. We therefore investigated the temporal patterns of functional outcome [using range of motion (ROM), American Knee Society (AKS) score, Western Ontario and McMaster Universities Arthritis Index (WOMAC) score, and 36-Item Short Form Health Survey (SF-36) score] over a 5-year period after uncomplicated TKA, and whether these patterns differed by implant type and patient age.
\end{abstract}

Materials and methods: This prospective study evaluated 138 patients who underwent unilateral TKA with either a mobile-bearing (MB) or fixed-bearing (FB) posterior-stabilized prosthesis. An independent investigator evaluated the functional outcome at five time points: preoperatively and at 6-month, 1-year, 2-year, and 5-year follow-up. Differences in functional outcomes between adjacent time points were evaluated by mixed-effect model repeat measurement (MMRM).

Results: The different functional outcome scores showed improvement till 6 months-2 years, followed by a variable decline. In patients aged $\geq 68$ years with an MB implant, most of the functional outcome scores declined between 2 and 5 years after variable initial improvement till 6 months -2 years, whereas the parameters plateaued after 2 years in those aged $<68$ years and in older patients with an FB implant.

Conclusions: A decline in function and pain relief occurs 2 years after TKA. This decline is more evident in older patients with an MB prosthesis. Based on these findings, we believe that use of MB implants in older patients ( $\geq 68$ years) requires further investigation.

Level of evidence: Level 3.

Keywords: Total knee arthroplasty, Functional outcome, Pattern, Age, Prosthesis

\section{Introduction}

Evidence-based knowledge about the temporal pattern of functional results after total knee arthroplasty (TKA) has far-reaching clinical implications. Long-term follow-up studies after TKA have shown survival rates of up to $97 \%$ at 15 and 20 years $[1,2]$. Survival rates provide important information to physicians and patients; however, it is equally important to know how well the knee will

\footnotetext{
*Correspondence: osktk2000@yahoo.com

1 Joint Reconstruction Center, Seoul National University Bundang Hospital, 82 Gumi-ro, 173 Beon-gil, Bundang-gu, Seongnam-si, Gyeonggi-do 13620, Republic of Korea

Full list of author information is available at the end of the article
}

function after TKA, in both the short and long term [3]. From the surgeon's perspective, knowing the timeline of the extent of recovery and its maintenance after uneventful TKA is important to counsel patients.

Literature provides contradicting information about the temporal pattern of improvement in functional outcome after TKA. Over the last three decades, during which TKA has been successfully performed and evolved, only a handful of studies have attempted to address this issue [3-13]. Most previous studies are limited by the fact that either only the clinician-examined Knee Society Score (KSS) was used for evaluation [4, 6, 7, 9] rather than patient-reported outcomes measures (PROMs), 
which are of real value to the patient, or the time points at which the patients were assessed were too spaced out, thereby potentially missing the true timeline $[5,8,12]$, or the number of patients available at final follow-up was too small [11], raising concerns about whether the findings are generally applicable to society. While some studies suggest that pain and function continue to improve for 4-7 years after TKA [5, 12], others suggest that these improvements are limited to 2 years after TKA, after which they begin to decline $[3,8]$. Although no evidence of the superiority of either fixed-bearing (FB) or mobilebearing (MB) types of implant has been found with respect to range of movement (ROM), pain, stiffness, or function [14], the rate of recovery may differ according to bearing type [15]. Furthermore, TKA performed at older patient age may affect the timeline of functional recovery, given the fact that such patients have a higher medical complication rate. Considering that we had prospectively collected data of a sizable number of patients at multiple time points by using various outcome scores up to midterm follow-up of 5 years, with both FB and MB types of implant, we believed that we were in a position to address these issues.

The aims of this study are: (1) to investigate the temporal patterns of functional outcomes [using ROM, American Knee Society Score [AKS], Western Ontario and McMaster Universities Arthritis Index (WOMAC) score, and 36-Item Short Form Health Survey (SF-36) score] over a 5-year period after uncomplicated TKA, and (2) to determine whether the temporal patterns of functional outcome after TKA differ by implant type and patient age.

\section{Patients and methods}

\section{Study design and setting}

All patients who underwent primary TKA at our tertiary care institution from November 2004 to May 2005 were prospectively evaluated for functional outcome at five time points from preoperative assessment until 5-year follow-up. This study was approved by the institutional review board of our hospital, and informed consent for use of medical information was obtained from all patients.

\section{Participants/study subjects}

Patients with osteoarthritis (OA) who underwent unilateral TKA during the study period and for whom outcome data were available at each of the five time points were included for analysis. During the study period, 177 patients underwent unilateral TKA at our institution and were assessed for eligibility. We excluded 23 patients on the basis of exclusion criteria and 8 patients on the basis of complications (Fig. 1). Moreover, complete follow-up data were not available for eight patients. Thus, 138 patients had complete 5-year follow-up data and were thus included in the analysis. Of the patients, 131 were female $(94.9 \%)$ and 7 were male $(5.1 \%)$, with mean [standard deviation (SD)] age of 67.7 (5.6) years. Their mean (SD) body weight, height, and body mass index were 62.6 (7.8) $\mathrm{kg}, 152.2(6.0) \mathrm{cm}$, and $26.9(3.0) \mathrm{kg} / \mathrm{m}^{2}$, respectively.

\section{Surgical protocol}

All TKAs were performed by a single surgeon using standard medial parapatellar arthrotomy with a tourniquet. One of two posteriorly stabilized prostheses [Genesis II (FB), Smith and Nephew, Memphis, USA or e.motion (MB), B. Braun Aesculap, Tuttlingen, Germany] was implanted for each patient. Implant selection was at surgeon discretion, without any preset selection criteria. For all cases, patellar resurfacing and cement fixation were performed. A standard postoperative rehabilitation protocol was used in all patients.

\section{Outcome measures}

Patients were evaluated by the same observer (one of the authors) at five time points, viz. preoperatively and at 6-month, 1-year, 2-year, and 5-year postoperative followup, by using ROM, AKS [16], WOMAC score [17], and SF-36 [18] score. The ROM of all patients was measured to the nearest $5^{\circ}$ using a standard $38-\mathrm{cm}$ clinical goniometer with the patient in supine position, calculated by subtracting the flexion contracture from maximum flexion.

\section{Statistical analysis}

All statistical analyses were conducted by using SPSS version 21.0 for Windows (SPSS Inc., Chicago, IL); $p$ value $<0.05$ was considered significant. Quantitative variables are expressed as mean and SD. Differences in functional outcome between adjacent time points were evaluated by mixed-effect model repeat measurement (MMRM). To determine whether temporal patterns of functional outcomes after TKA differed by implant type and patient age, we created four groups of patients as follows: (1) patients aged $<68$ years with an FB implant $(n=31)$, (2) patients aged $<68$ years with an MB implant $(n=32)$, (3) patients aged $\geq 68$ years with an FB implant $(n=38)$, and (4) patients aged $\geq 68$ years with an MB implant $(n=37)$. The number of patients aged $<68$ and $\geq 68$ years was 63 and 75, respectively. Sixty-nine patients had an FB implant, while another 69 patients had an MB implant. The baseline demographic data of the two age groups and the two implant groups of patients were comparable (Tables 1 and 2). In the four groups, the functional outcome scores between adjacent time points were compared by MMRM. In comparing the functional outcomes, 


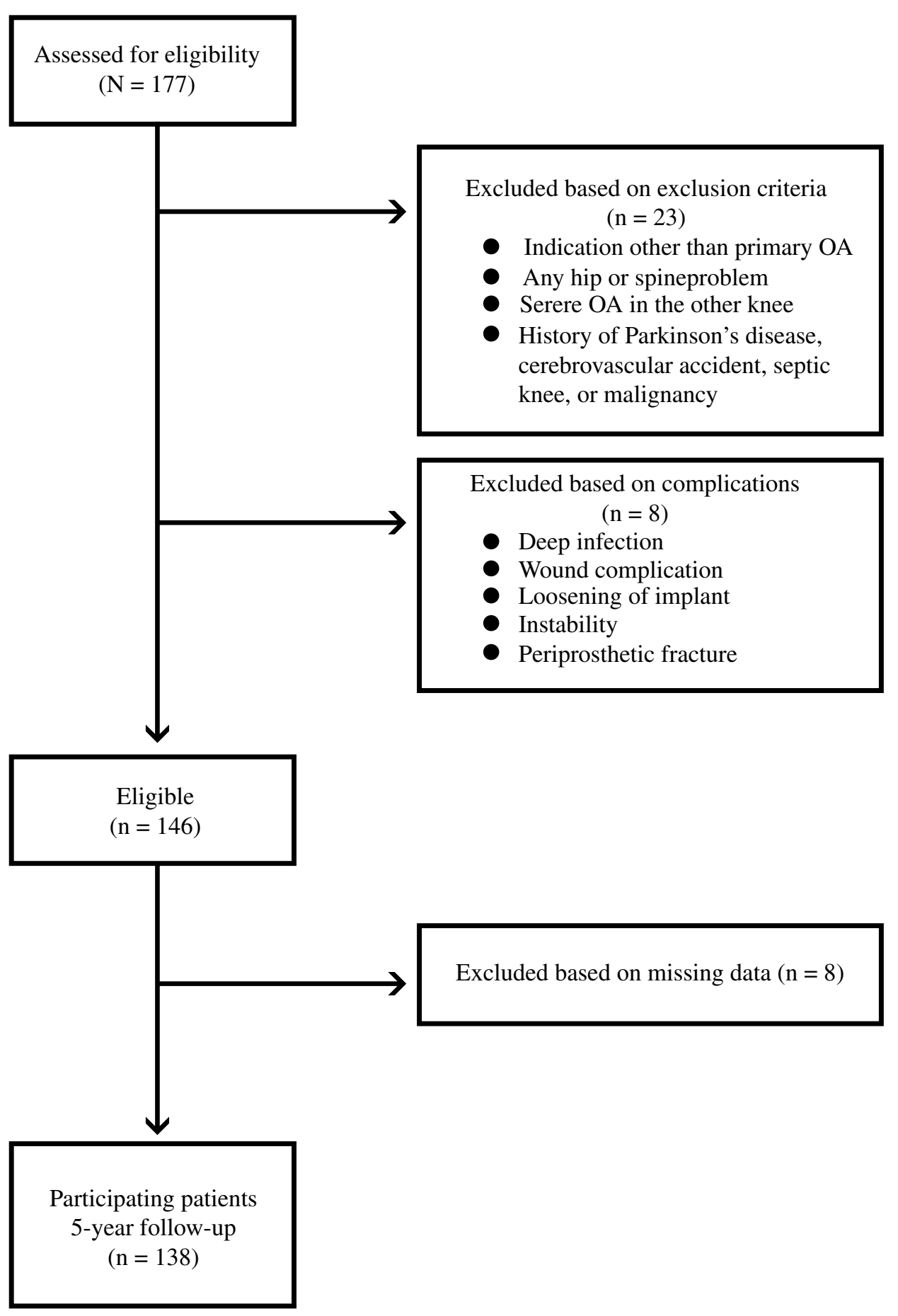

Fig. 1 Flowchart showing details of patient enrollment and follow-up

we considered a difference of $5^{\circ}$ in ROM and $6 \%$ in the outcome scales to be clinically important, as the motion arc was measured to the nearest $5^{\circ}$ and a $6 \%$ difference in maximum score has been suggested as the minimal clinically important difference for the WOMAC score and SF-36 score [19]. 
Table 1 Demographic data of patients in the two age groups

\begin{tabular}{lccr}
\hline Variable & $\begin{array}{l}<68 \text { years old } \\
(\boldsymbol{n}=\mathbf{6 3})\end{array}$ & $\begin{array}{l}\mathbf{2 6 8} \text { years old } \\
(\boldsymbol{n}=\mathbf{7 5})\end{array}$ & $\boldsymbol{p}$-Value \\
\hline Sex (female) & $61(96.8 \%)$ & $70(93.3 \%)$ & \\
Age (years) & $62.8(3.8)$ & $71.7(3.2)$ & $<0.001$ \\
Height $(\mathrm{cm})$ & $153.3(5.4)$ & $151.3(6.4)$ & 0.063 \\
Weight $(\mathrm{kg})$ & $63.5(6.7)$ & $61.8(8.7)$ & 0.210 \\
BMl $\left(\mathrm{kg} / \mathrm{m}^{2}\right)$ & $26.9(2.8)$ & $27.0(3.2)$ & 0.911 \\
\hline
\end{tabular}

Data presented as mean (SD) unless otherwise specified

$B M /$ body mass index

Table 2 Demographic data of patients in the two implant groups

\begin{tabular}{lccc}
\hline & MB $(\boldsymbol{n}=\mathbf{6 9})$ & FB $(\boldsymbol{n}=\mathbf{6 9})$ & $\boldsymbol{p}$-Value \\
\hline Sex (female) & $64(92.8 \%)$ & $67(97.1 \%)$ & \\
Age (years) & $66.8(4.8)$ & $68.5(6.2)$ & 0.069 \\
Height $(\mathrm{cm})$ & $152.3(6.4)$ & $152.1(5.7)$ & 0.831 \\
Weight $(\mathrm{kg})$ & $62.4(7.8)$ & $62.7(7.9)$ & 0.802 \\
BMl $\left(\mathrm{kg} / \mathrm{m}^{2}\right)$ & $26.9(2.9)$ & $27.0(3.1)$ & 0.866 \\
\hline
\end{tabular}

Data presented as mean (SD) unless otherwise specified

$M B$ mobile bearing, $F B$ fixed bearing, $B M I$ body mass index

\section{Results}

The different functional outcome scores showed improvement up to 6 months-2 years, after which most of the parameters declined (Table 3 ). Before operation, the mean flexion contracture was $12^{\circ}$ with further flexion of up to $139^{\circ}\left(\mathrm{ROM}, 127.2^{\circ}\right)$. The mean ROM showed improvement at 6 months $\left(133.8^{\circ}\right)$ and was constant after that till 1 year $\left(134.2^{\circ}\right)$, before demonstrating a decline at
2 years $\left(133.2^{\circ}\right)$ and 5 years $\left(130.2^{\circ}\right)$, although the decline was not clinically important (Fig. 2a). The mean AKS knee score showed improvement at 6 months, a small decline at 1 year, then improvement again at 2 years, reaching the 6-month level. However, it again demonstrated a decline between 2 and 5 years (Fig. 2b). The mean AKS functional score continuously improved until 2 years and was then constant until 5 years (Fig. 2c). The mean WOMAC pain score showed a significant improvement in pain until 1 year, a plateau until 2 years, then a marked decline between 2 and 5 years (Fig. 2d). The mean WOMAC stiffness score improved until 1 year then demonstrated a plateau phase until 5 years (Fig. 2e). The mean WOMAC function score showed improvement until 1 year and was then constant until 2 years, before declining between 2 and 5 years (Fig. 2f). The mean SF-36 physical component summary (PCS) score improved from baseline until 1 year and was constant until 2 years, before showing a decline at 5 years (Fig. 2g). The mean SF-36 mental component summary (MCS) score improved from baseline at 6 months, was constant until 2 years, then showed a marked decline between 2 and 5 years (Fig. 2h).

Most of the functional outcome scores declined between 2 and 5 years in patients aged $\geq 68$ years with an $\mathrm{MB}$ implant, after a variable initial improvement for 6 months to 2 years, whereas most of the parameters plateaued after 2 years in those aged $<68$ years and in the older patients with an FB implant. In patients aged $<68$ years with an FB implant, the AKS knee score, WOMAC pain score, WOMAC stiffness score, and WOMAC function score improved at 6 months and were constant thereafter until 5 years, whereas the AKS function score and SF-36 PCS showed improvement

Table 3 Temporal patterns of functional outcome scales during the 5-year period

\begin{tabular}{|c|c|c|c|c|c|c|c|c|c|}
\hline Score & Preop. & $p$-Value & PO 6 months & $p$-Value & PO 1 year & $p$-Value & PO 2 years & $p$-Value & PO 5 years \\
\hline $\mathrm{ROM}$ & $127.2(1.4)$ & $<0.001$ & $133.8(0.7)$ & 0.436 & $134.2(0.8)$ & 0.043 & $133.2(1.0)$ & $<0.001$ & $130.2(1.0)$ \\
\hline \multicolumn{10}{|l|}{ AKS } \\
\hline Knee score (100) & $44.4(0.7)$ & $<0.001$ & $95.2(0.5)$ & 0.001 & $93.5(0.5)$ & 0.001 & $95.0(0.5)$ & $<0.001$ & $92.5(0.6)$ \\
\hline Function score (100) & $58.7(1.0)$ & $<0.001$ & $91.3(0.9)$ & $<0.001$ & $96.3(0.8)$ & 0.040 & $97.7(0.6)$ & 0.186 & $96.4(0.9)$ \\
\hline \multicolumn{10}{|l|}{ WOMAC } \\
\hline Pain (20) & $11.4(0.3)$ & $<0.001$ & $3.0(0.3)$ & 0.012 & $2.2(0.2)$ & 0.329 & $1.9(0.3)$ & 0.002 & $3.2(0.4)$ \\
\hline Stiffness (8) & $4.8(0.2)$ & $<0.001$ & $2.3(0.1)$ & $<0.001$ & $1.7(0.1)$ & 0.192 & $1.5(0.1)$ & 0.166 & $1.8(0.2)$ \\
\hline Function (68) & $39.5(1.0)$ & $<0.001$ & $18.7(0.9)$ & $<0.001$ & $14.4(0.8)$ & 0.242 & $13.5(0.8)$ & 0.002 & $17.7(1.3)$ \\
\hline \multicolumn{10}{|l|}{ SF-36 } \\
\hline PCS & $29.3(0.5)$ & $<0.001$ & $41.3(0.7)$ & $<0.001$ & $45.4(0.6)$ & 0.356 & $44.6(0.7)$ & 0.002 & $42.0(0.8)$ \\
\hline MCS & $42.0(1.0)$ & 0.001 & $46.8(1.0)$ & 0.086 & $48.9(0.9)$ & 0.854 & $49.1(1.0)$ & 0.002 & $44.8(1.2)$ \\
\hline
\end{tabular}

Preop. preoperative, PO postoperative, ROM range of movement, AKS American Knee Society score, WOMAC Western Ontario and McMaster Universities Arthritis Index score, SF-36 36-Item Short Form Health Survey, PCS physical component summary, MCS mental component summary 


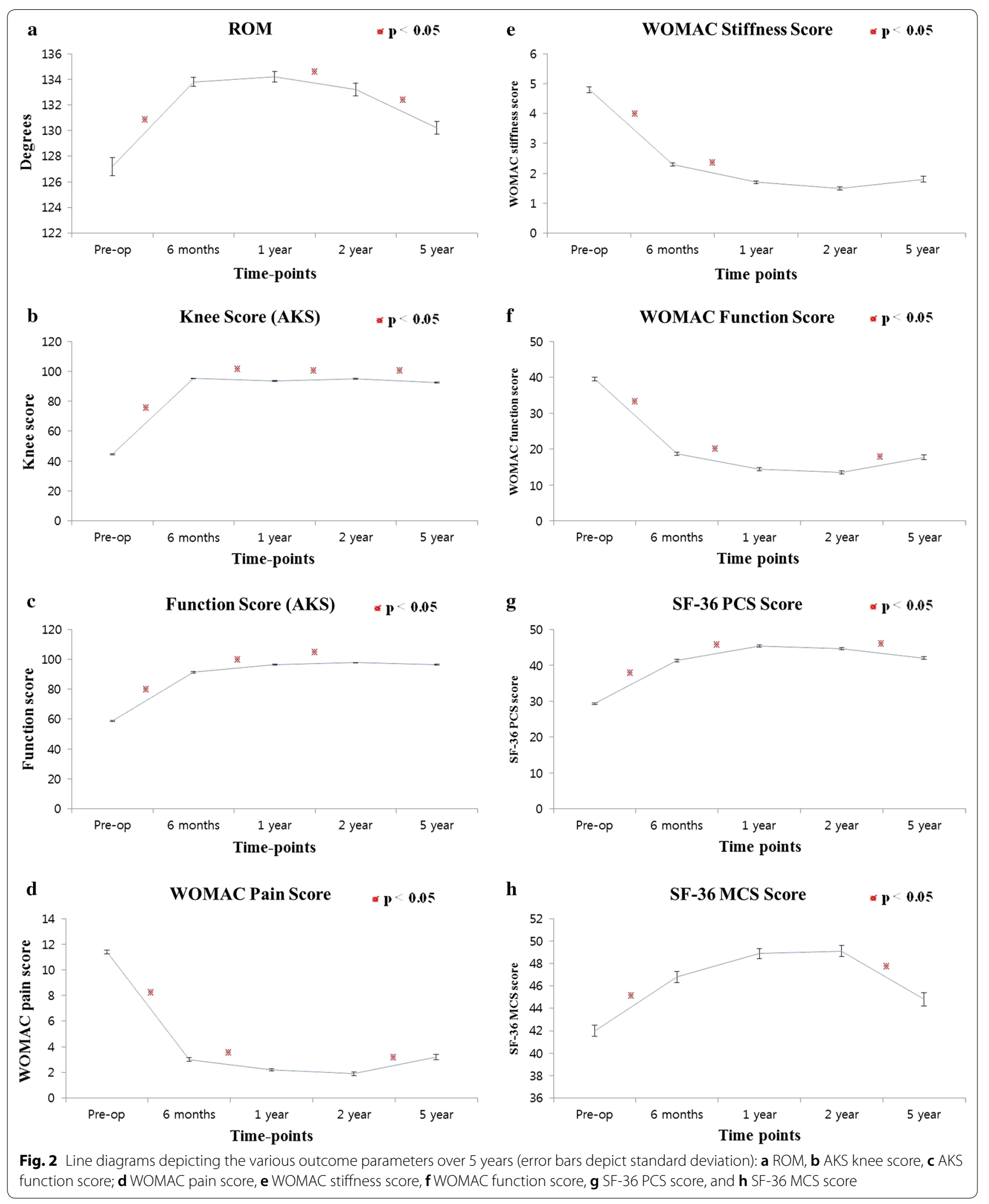


until 1 year before becoming constant up to 5 years (Table 4). The SF-36 MCS score tended to improve at 6 months $(p=0.062)$, after which it showed a constant value until 5 years. ROM did not show any improvement from baseline until 5 years. In the patients aged $<68$ years with an MB implant, the ROM, AKS knee score, WOMAC stiffness score, and SF-36 MCS score improved at 6 months, whereas the AKS function score improved until 2 years and the WOMAC pain score, WOMAC function score, and SF-36 MCS showed improvement until 1 year. Except for ROM, which declined, and the WOMAC function score, which showed a tendency to decline $(p=0.092)$, all other scores were constant between 2 and 5 years (Table 5 ). In patients aged $\geq 68$ years with an FB implant, ROM and
AKS function scores improved at 6 months, whereas the WOMAC stiffness score improved until 1 year, and the AKS knee score, WOMAC pain score, WOMAC function score, and SF-36 PCS showed improvement until 2 years. The SF-36 MCS score, on the other hand, did not show any improvement from baseline until 5 years. The ROM and SF-36 PCS score declined, and the AKS knee score $(p=0.082)$ and WOMAC function score $(p=0.088)$ showed a tendency to decline between 2 and 5 years (Table 6 ). In patients aged $\geq 68$ years with an MB implant, the ROM, AKS knee score, WOMAC pain score, and SF-36 MCS score improved at 6 months, whereas the AKS function score, WOMAC function score, and SF-36 PCS score improved until 1 year, and the WOMAC stiffness score improved until 2 years. All

Table 4 Temporal pattern of functional outcome scales in patients aged < 68 years with an FB implant

\begin{tabular}{|c|c|c|c|c|c|c|c|c|c|}
\hline Score & Preop. & $p$-Value & PO 6 months & $p$-Value & PO 1 year & $p$-Value & PO 2 years & $p$-Value & PO 5 years \\
\hline $\mathrm{ROM}$ & $134.2(2.1)$ & 0.603 & 135.5 (1.9) & 0.231 & $133.9(2.5)$ & 0.547 & $134.5(2.8)$ & 0.071 & $132.9(2.5)$ \\
\hline \multicolumn{10}{|l|}{ AKS } \\
\hline Knee score (100) & $48.1(1.2)$ & $<0.001$ & $95.8(0.9)$ & 0.209 & $94.6(1.2)$ & 0.615 & $95.1(1.1)$ & 0.069 & $92.1(1.7)$ \\
\hline Function score (100) & $58.6(2.2)$ & $<0.001$ & $92.9(1.7)$ & $<0.001$ & $99.3(0.5)$ & 0.758 & $99.0(0.9)$ & 0.891 & $99.1(0.9)$ \\
\hline \multicolumn{10}{|l|}{ WOMAC } \\
\hline Pain (20) & $12.3(0.7)$ & $<0.001$ & $2.5(0.5)$ & 0.212 & $1.6(0.5)$ & 0.852 & $1.5(0.4)$ & 0.336 & $2.3(0.7)$ \\
\hline Stiffness (8) & $4.8(0.4)$ & $<0.001$ & $2.0(0.3)$ & 0.122 & $1.4(0.2)$ & 0.653 & $1.6(0.2)$ & 0.916 & $1.6(0.4)$ \\
\hline Function (68) & $39.6(2.3)$ & $<0.001$ & $13.4(1.9)$ & 0.342 & $11.9(1.7)$ & 0.780 & $11.5(1.4)$ & 0.673 & $12.6(2.6)$ \\
\hline \multicolumn{10}{|l|}{ SF-36 } \\
\hline PCS & $31.2(1.0)$ & $<0.001$ & $44.6(1.7)$ & 0.048 & $48.1(1.1)$ & 0.436 & $46.8(1.3)$ & 0.272 & $44.5(1.9)$ \\
\hline MCS & $39.8(2.4)$ & 0.062 & $45.7(2.6)$ & 0.484 & $48.2(1.9)$ & 0.798 & $49.0(2.2)$ & 0.152 & $44.1(2.2)$ \\
\hline
\end{tabular}

FB fixed bearing, Preop. preoperative, $P O$ postoperative, ROM range of movement, AKS American Knee Society score, WOMACWestern Ontario and McMaster Universities Arthritis Index score, SF-36 36-Item Short Form Health Survey, PCS physical component summary, MCS mental component summary

Italics indicates significant $p$-values

Table 5 Temporal pattern of functional outcome scales in patients aged < 68 years with an MB implant

\begin{tabular}{|c|c|c|c|c|c|c|c|c|c|}
\hline Score & Preop. & $p$-Value & PO 6 months & $p$-Value & PO 1 year & $p$-Value & PO 2 years & $p$-Value & PO 5 years \\
\hline $\mathrm{ROM}$ & $127.0(2.9)$ & 0.022 & $133.3(1.2)$ & 0.198 & $134.4(1.3)$ & 0.017 & $132.2(1.5)$ & 0.001 & $128.3(1.6)$ \\
\hline \multicolumn{10}{|l|}{ AKS } \\
\hline Knee score (100) & $44.1(1.9)$ & $<0.001$ & $94.6(1.2)$ & 0.003 & $91.2(1.2)$ & 0.006 & $93.7(0.9)$ & 0.671 & $93.4(1.1)$ \\
\hline Function score (100) & $59.7(2.7)$ & $<0.001$ & $95.0(1.8)$ & 0.722 & $95.6(1.6)$ & 0.022 & $98.1(1.1)$ & 0.521 & $98.1(1.1)$ \\
\hline \multicolumn{10}{|l|}{ WOMAC } \\
\hline Pain (20) & $10.6(0.9)$ & $<0.001$ & $3.9(0.7)$ & 0.014 & $2.4(0.4)$ & 0.806 & $2.3(0.4)$ & 0.718 & $2.1(0.5)$ \\
\hline Stiffness (8) & $4.6(0.4)$ & $<0.001$ & $2.4(0.3)$ & 0.121 & $2.0(0.3)$ & 0.589 & $1.8(0.2)$ & 0.171 & $1.3(0.2)$ \\
\hline Function (68) & $38.2(2.6)$ & $<0.001$ & $22.4(2.0)$ & $<0.001$ & $13.4(1.2)$ & 0.231 & $15.4(1.4)$ & 0.092 & $11.4(2.1)$ \\
\hline \multicolumn{10}{|l|}{ SF-36 } \\
\hline PCS & $29.2(1.3)$ & $<0.001$ & $39.9(1.6)$ & 0.001 & $46.6(1.2)$ & 0.023 & $43.2(1.3)$ & 0.279 & $45.3(1.2)$ \\
\hline MCS & $41.8(1.5)$ & 0.002 & $47.0(1.9)$ & 0.340 & $48.9(1.5)$ & 0.314 & $46.8(1.8)$ & 0.193 & $50.3(1.8)$ \\
\hline
\end{tabular}

$M B$ mobile bearing, Preop. preoperative, $P O$ postoperative, ROM range of movement, AKS American Knee Society score, WOMACWestern Ontario and McMaster Universities Arthritis Index score, SF-36 36-Item Short Form Health Survey, PCS physical component summary, MCS mental component summary 
Table 6 Temporal pattern of functional outcome scales in patients aged $\geq 68$ years with an FB implant

\begin{tabular}{|c|c|c|c|c|c|c|c|c|c|}
\hline Score & Preop. & $p$-Value & PO 6 months & $p$-Value & PO 1 year & $p$-Value & PO 2 years & $p$-Value & PO 5 years \\
\hline $\mathrm{ROM}$ & $122.6(2.6)$ & 0.001 & $133.0(1.3)$ & 0.313 & $134.0(1.3)$ & 0.022 & $132.0(1.3)$ & $<0.001$ & $127.5(1.8)$ \\
\hline \multicolumn{10}{|l|}{ AKS } \\
\hline Knee score (100) & $43.6(1.2)$ & $<0.001$ & $94.7(0.9)$ & 0.708 & $94.3(0.8)$ & 0.010 & $96.7(0.6)$ & 0.082 & $94.9(0.8)$ \\
\hline Function score (100) & $59.2(1.7)$ & $<0.001$ & $88.7(1.7)$ & 0.173 & $91.6(2.1)$ & 0.517 & $93.0(1.6)$ & 0.367 & $95.3(1.7)$ \\
\hline \multicolumn{10}{|l|}{ WOMAC } \\
\hline Pain (20) & $11.2(0.5)$ & $<0.001$ & $2.9(0.5)$ & 0.420 & $3.3(0.5)$ & 0.015 & $2.0(0.5)$ & 0.177 & $3.4(0.8)$ \\
\hline Stiffness (8) & $4.8(0.3)$ & $<0.001$ & $2.4(0.2)$ & 0.014 & $1.6(0.2)$ & 0.842 & $1.6(0.2)$ & 0.484 & $1.9(0.4)$ \\
\hline Function (68) & $40.6(1.8)$ & $<0.001$ & $19.9(1.6)$ & 0.968 & $19.9(1.7)$ & 0.013 & $16.2(1.5)$ & 0.088 & $21.3(2.9)$ \\
\hline \multicolumn{10}{|l|}{ SF-36 } \\
\hline PCS & $27.5(0.7)$ & $<0.001$ & $39.2(1.2)$ & 0.043 & $41.8(1.1)$ & 0.027 & $45.1(1.4)$ & 0.001 & $36.5(2.2)$ \\
\hline MCS & $45.5(1.9)$ & 0.652 & $46.5(1.9)$ & 0.855 & $46.9(1.9)$ & 0.874 & $47.1(2.0)$ & 0.171 & $43.2(2.6)$ \\
\hline
\end{tabular}

FB fixed bearing, Preop. preoperative, $P O$ postoperative, ROM range of movement, AKS American Knee Society score, WOMACWestern Ontario and McMaster Universities Arthritis Index score, SF-36 36-Item Short Form Health Survey, PCS physical component summary, MCS mental component summary

Italics indicates significant $p$-values

Table 7 Temporal pattern of functional outcome scales in patients aged $\geq 68$ years with an MB implant

\begin{tabular}{|c|c|c|c|c|c|c|c|c|c|}
\hline Score & Preop. & $p$-Value & PO 6 months & $p$-Value & PO 1 year & $p$-Value & PO 2 years & $p$-Value & PO 5 years \\
\hline ROM & $126.2(2.8)$ & 0.003 & 133.5 & 0.362 & $134.3(1.6)$ & 0.900 & $134.2(1.9)$ & 0.027 & $132.0(2.2)$ \\
\hline \multicolumn{10}{|l|}{ AKS } \\
\hline Knee score (100) & $42.5(1.3)$ & $<0.001$ & $95.8(1.1)$ & 0.052 & $93.9(1.0)$ & 0.852 & $94.0(1.1)$ & $<0.001$ & $90.1(1.2)$ \\
\hline Function score (100) & $57.6(2.4)$ & $<0.001$ & $89.5(2.0)$ & $<0.001$ & $98.9(0.8)$ & 0.944 & $98.8(0.8)$ & 0.837 & $95.2(2.4)$ \\
\hline \multicolumn{10}{|l|}{ WOMAC } \\
\hline Pain (20) & $11.4(0.6)$ & $<0.001$ & $2.6(0.5)$ & 0.069 & $1.5(0.3)$ & 0.297 & $2.2(0.6)$ & $<0.001$ & $4.3(0.8)$ \\
\hline Stiffness (8) & $5.0(0.3)$ & $<0.001$ & $2.4(0.2)$ & 0.013 & $1.8(0.2)$ & 0.035 & $1.2(0.2)$ & 0.001 & $2.3(0.3)$ \\
\hline Function (68) & $39.1(1.6)$ & $<0.001$ & $18.6(1.3)$ & $<0.001$ & $12.4(1.3)$ & 0.612 & $11.6(1.4)$ & $<0.001$ & $21.0(2.0)$ \\
\hline \multicolumn{10}{|l|}{ SF-36 } \\
\hline PCS & $29.5(0.8)$ & $<0.001$ & $42.0(1.4)$ & 0.013 & $45.8(1.4)$ & 0.442 & $44.7(1.3)$ & 0.016 & $40.7(1.2)$ \\
\hline MCS & $40.6(1.8)$ & 0.003 & $47.7(1.9)$ & 0.126 & $51.4(1.6)$ & 0.427 & $52.6(1.5)$ & $<0.001$ & $43.4(2.0)$ \\
\hline
\end{tabular}

MB mobile bearing, Preop. preoperative, PO postoperative, ROM range of movement, AKS American Knee Society score, WOMACWestern Ontario and McMaster Universities Arthritis Index score, SF-36 36-Item Short Form Health Survey, PCS physical component summary, MCS mental component summary

Italics indicates significant $p$-values

scores except the AKS function score declined between 2 and 5 years (Table 7$)$.

\section{Discussion}

Literature provides contradicting information regarding the temporal pattern of improvement in functional outcome after TKA. This uncertainty is further compounded by the fact that different outcome measurement tools are known to produce different results and have different capabilities [20]. Using generic, diseasespecific, and performance-based measurements is therefore recommended to fully appreciate recovery after knee replacement $[20,21]$. We prospectively evaluated functional outcome after uncomplicated TKA at various time points over 5 years using three different outcome measures, encompassing clinician- and patient-reported tools, besides ROM. We also identified differences, if any, in functional outcome after TKA over the same time points between $\mathrm{FB}$ and $\mathrm{MB}$ prostheses, and between patients aged $\geq 68$ years as compared with their younger counterparts.

We found that most of the functional outcome parameters improved for 6 months to 2 years, followed by a downward trend between 2 and 5 years after uncomplicated TKA. Some previous studies have suggested that pain and function continue to improve for 4-7 years after TKA $[5,12]$. A prospective study evaluating 49 total hip arthroplasty (THA)/TKA patients found a significant improvement in the WOMAC score and some components of the SF-36 score between 6 months and 7 years [5]. However, those authors did not study the outcome parameters between 6 months and 7 years, and this 
large gap in the timeline may mask important trends in the results, which could have important clinical implications. In our study, we evaluated the parameters at relatively short time intervals to address this limitation. Another study, evaluating perceived physical functioning in 44 THA/TKA patients by using the Hip Disability and Osteoarthritis Outcome Score (HOOS) and the Knee Injury and Osteoarthritis Outcome Score (KOOS) questionnaires, found a more significant improvement at 4 years than at 6 months [12]. However, no objective and clinician-reported outcome measures were used in that study. Moreover, the sample size was too small to make any definite conclusions, and the outcome was evaluated at fewer time points. We assessed functional outcome by using various outcome measurement tools, encompassing clinician- and patient-reported scores.

A few studies in literature suggest that improvements in functional parameters are limited to 1-2 years after TKA, after which they begin to decline $[3,8]$. A prospective study, evaluating health-related quality of life (HRQOL) in 102 consecutive TKA patients by using the KOOS and SF-36 scores, found that the maximum improvement was evident at 1 year, after which the scores declined until 5 years [8]. However, that was a questionnaire-based study and did not consider objective clinical parameters. In our study, which assessed functional outcome using objective parameters, we also observed a decline in most scores between 2 and 5 years. Another study, evaluating functional results in 50 TKA patients by using KOOS and ROM measurements, reported that most of the improvements in pain and function were achieved by 6 months, with further small improvements up to 2 years before a small decline at 4 years [3]. Our findings closely resemble those of previous studies, while assessing function more elaborately and incorporating more patients. Although painful patellar clunk or crepitation (PCC) has been reported as one of the causes for late-onset pain after TKA, especially with contemporary posterior-stabilized prosthesis designs, no patients with an e.motion implant and only one patient with a Genesis II were diagnosed with PCC in a retrospective study of 948 primary posterior-stabilized TKAs [22]. Furthermore, the implant design of the two prostheses, including the shapes of the patellar component and trochlear groove, have not been specifically reported to be linked with knee pain or decreased ROM after TKA, thus ruling out implant-related bias in the decline of functional scores observed in this study.

We observed that the temporal pattern of functional outcome after TKA differed according to both implant type and patient age. Previously published studies on the effect of implant type (FB or MB) on the evolution of functional outcome after TKA could not find any such differences between the two implant groups [14, 23]. The theoretical advantage of MB TKA designs, due to increased congruity and unconstrained mobility of the bearing, has not been translated into improved long-term clinical outcomes or implant survivorship. A meta-analysis of the two TKA designs did not find any significant difference in clinical or radiological outcomes and complication rates between fixed- and mobile-bearing TKAs [14]. However, the above-mentioned study did not consider various time points in the recovery after TKA, which has important clinical implications. In our study, we evaluated the functional outcome after TKA in the two groups at various time points, to comprehensively evaluate patterns of recovery. A randomized controlled clinical trial comparing the outcomes of $\mathrm{FB}$ and $\mathrm{MB}$ TKAs found that most of the gait parameters improved at 5 years after TKAs using FB implants in older patients ( $>70$ years) and for TKAs using MB implants in younger patients [15]. However, their sample size was relatively small; moreover, they did not comprehensively study the temporal pattern of recovery in the various subgroups. We used MMRM to study the pattern of recovery in functional outcome at various time points, which has been reported to be a superior approach for controlling type I error rates and minimizing biases [24]. MB TKA designs have evolved over time, based on the range of freedom of the polyethylene insert, from the initial meniscal design to the rotating platform type, finally leading to the development of the anteroposterior glide platform variety. However, in a retrospective comparative study of the three types of mobile bearings, no difference in clinical or radiological results or implant survivorship was reported [25].

Only limited information is available in literature regarding comparison of the temporal pattern of functional outcome after TKA in older versus young patients. A prospective study comparing functional outcome and pain after TKA between patients aged $\leq 55$ years and $>55$ years at time of index surgery found no significant difference in pain or knee performance over 10 years of follow-up between the two groups. The authors, however, found higher overall function in the younger age group throughout the study period [26]. However, they did not evaluate the temporal pattern of recovery in the two groups at various time points. In our study, which had a relatively larger sample size, we found a decline in function between 2 and 5 years in the older patients ( $\geq 68$ years) when implanted with an MB prosthesis. Considering the greater degree of freedom of movement provided by an MB implant compared with a fixed-bearing one, full function of the former implant appears to be more dependent on adequate quadriceps muscle power. The decline in functional outcome seen in older patients 
with an MB implant may be explained by decreased muscle strength associated with aging in such patients.

This study has a number of strengths, including use of a standardized clinical pathway, use of validated outcome measures, and 5-year follow-up. However, there are some limitations to this study, which should be considered when interpreting the results. First, we did not have a control group with which to compare the changes in functional outcome over the 5-year period. However, in an extensive literature review, we could not find any longterm prospective study that evaluated such outcome measures in a healthy population. Therefore, the above results can be assumed to be relevant to the general population. Second, as we only included unilateral TKAs in our study, effects of OA of the other knee on the serial outcome measures cannot be ruled out. However, to reduce this ambiguity, we excluded patients with severe OA of the other knee from analysis in the study. Moreover, this study provides empirical data that lay the path for future studies on bilateral TKA. Third, the lack of randomization regarding the type of prosthesis (FB or $\mathrm{MB}$ ) used precludes the abolition of an inherent selection bias in the study. This should be borne in mind when interpreting the results. The use of a single type of $\mathrm{FB}$ or $\mathrm{MB}$ implant for all patients is another limitation that requires consideration, as this could be a potential source of selection bias.

In conclusion, a decline in function and pain relief occurs after 2 years following TKA. This decline is more evident in older patients ( $\geq 68$ years) and in those with a MB prosthesis. Patients should be counseled preoperatively about the anticipated decline in function over time after 2 years following TKA. Based on these findings, we believe that use of MB implants in older patients ( $\geq 68$ years) should be questioned. We further recommend future studies with larger sample size and longer follow-up to comprehensively evaluate the effect of $\mathrm{MB}$ implants in older patients.

\begin{abstract}
Authors' contribution
$V T, J L, G S, Y G K$ did data collection, participated in data analysis and drafted the manuscript. TKK conceptualized the study, participated in data analysis and drafted the manuscript. All authors read and approved the final manuscript.
\end{abstract}

\section{Author details}

1 Joint Reconstruction Center, Seoul National University Bundang Hospital, 82 Gumi-ro, 173 Beon-gil, Bundang-gu, Seongnam-si, Gyeonggi-do 13620, Republic of Korea. ${ }^{2}$ Department of Orthopaedic Surgery, Seoul National University College of Medicine, Seoul, Republic of Korea.

\section{Acknowledgements}

This work was performed at the Joint Reconstruction Center, Seoul National University Bundang Hospital.

\section{Competing interests}

Each author certifies that he or she, or a member of his or her immediate family, has no funding or commercial associations (e.g., consultancies, stock ownership, equity interest, patent/licensing arrangements, etc.) that might pose a conflict of interest in connection with the submitted article. The authors declare that they have no competing interests.

\section{Ethics approval and consent to participate}

All procedures performed in studies involving human participants were in accordance with the ethical standards of the institutional and/or national research committee and with the 1964 Helsinki Declaration and its later amendments or comparable ethical standards. Each author certifies that his or her institution has approved the human protocol for this investigation, that all investigations were conducted in conformity with ethical principles of research, and that informed consent was obtained.

\section{Funding}

This research did not receive any specific grant from funding agencies in the public, commercial, or not-for-profit sectors.

\section{Informed consent}

Informed consent was obtained from all individual participants included in the study.

\section{Publisher's Note}

Springer Nature remains neutral with regard to jurisdictional claims in published maps and institutional affiliations.

Received: 22 June 2017 Accepted: 31 January 2019

Published online: 25 March 2019

\section{References}

1. Callaghan JJ, Wells CW, Liu SS, Goetz DD, Johnston RC (2010) Cemented rotating-platform total knee replacement: a concise follow-up, at a minimum of twenty years, of a previous report. J Bone Joint Surg Am 92(7):1635-1639

2. Rodriguez JA, Bhende H, Ranawat CS (2001) Total condylar knee replacement: a 20-year followup study. Clin Orthop Relat Res 388:10-17

3. Nerhus TK, Heir S, Thornes E, Madsen JE, Ekeland A (2010) Time-dependent improvement in functional outcome following LCS rotating platform knee replacement. Acta Orthop 81(6):727-732

4. Benjamin J, Johnson R, Porter S (2003) Knee scores change with length of follow-up after total knee arthroplasty. J Arthroplasty 18(7):867-871

5. Bruyère $O$, Ethgen $O$, Neuprez $A$, Zégels $B$, Gillet $P$, Huskin JP, Reginster JY (2012) Health-related quality of life after total knee or hip replacement for osteoarthritis: a 7-year prospective study. Arch Orthop Trauma Surg 132(11):1583-1587

6. Konig A, Scheidler M, Rader C, Eulert J (1997) The need for a dual rating system in total knee arthroplasty. Clin Orthop Relat Res 345:161-167

7. Meding JB, Meding LK, Ritter MA, Keating EM (2012) Pain relief and functional improvement remain 20 years after knee arthroplasty. Clin Orthop Relat Res 470(1):144-149

8. Nilsdotter AK, Toksvig-Larsen S, Roos EM (2009) A 5 year prospective study of patient-relevant outcomes after total knee replacement. Osteoarthritis Cartilage 17(5):601-606

9. Ritter MA, Thong AE, Davis KE, Berend ME, Meding JB, Faris PM (2004) Long-term deterioration of joint evaluation scores. J Bone Joint Surg $\mathrm{Br}$ 86(3):438-442

10. Seng C, Yeo SJ, Wee JL, Subanesh S, Chong HC, Lo NN (2011) Improved clinical outcomes after high-flexion total knee arthroplasty: a 5-year follow-up study. J Arthroplasty 26(7):1025-1030

11. van der Linden ML, Rowe PJ, Myles CM, Burnett R, Nutton RW (2007) Knee kinematics in functional activities seven years after total knee arthroplasty. Clin Biomech 22(5):537-542

12. Vissers MM, Bussmann JB, de Groot IB, Verhaar JA, Reijman M (2013) Physical functioning four years after total hip and knee arthroplasty. Gait Posture 38(2):310-315

13. Williams DP, Blakey CM, Hadfield SG, Murray DW, Price AJ, Field RE (2013) Long-term trends in the Oxford knee score following total knee replacement. Bone Joint J 95-B(1):45-51

14. Smith TO, Ejtehadi F, Nichols R, Davies L, Donell ST, Hing CB (2010) Clinical and radiological outcomes of fixed-versus mobile-bearing total knee 
replacement: a meta-analysis. Knee Surg Sports Traumatol Arthrosc 18(3):325-340

15. Jolles BM, Grzesiak A, Eudier A, Dejnabadi H, Voracek C, Pichonnaz C Aminian K, Martin E (2012) A randomised controlled clinical trial and gait analysis of fixed-and mobile-bearing total knee replacements with a fiveyear follow-up. J Bone Joint Surg Br 94(5):648-655

16. Insall JN, Dorr LD, Scott RD, Scott WN (1989) Rationale of the Knee Society clinical rating system. Clin Orthop Relat Res 248:13-14

17. Bellamy N, Buchanan WW, Goldsmith CH, Campbell J, Stitt LW (1988) Validation study of WOMAC: a health status instrument for measuring clinically important patient relevant outcomes to antirheumatic drug therapy in patients with osteoarthritis of the hip or knee. J Rheumatol 15(12):1833-1840

18. Ware JE Jr, Sherbourne CD (1992) The MOS 36-item short-form health survey (SF-36). I. Conceptual framework and item selection. Med Care 30(6):473-483

19. Angst F, Aeschlimann A, Stucki G (2001) Smallest detectable and minimal clinically important differences of rehabilitation intervention with their implications for required sample sizes using WOMAC and SF-36 quality of life measurement instruments in patients with osteoarthritis of the lower extremities. Arthritis Rheum 45(4):384-391

20. van Hemert WLW, Meyers WGH, Kleijn LLA, Heyligers IC, Grimm B (2009) Functional outcome of knee arthroplasty is dependent upon the evaluation method employed. Eur J Orthop Surg Traumatol 19(6):415-422

21. Torres-Claramunt R, Leal J, Hinarejos P, Pelfort X, Puig L (2013) Correlation study between KSS, WOMAC and SF-36 scores in patients undergoing total knee arthroplasty in a Spanish speaking population. J Arthroplasty 28(6):950-953

22. Choi WC, Ryu KJ, Lee S, Seong SC, Lee MC (2013) Painful patellar clunk or crepitation of contemporary knee prostheses. Clin Orthop Relat Res 471(5):1512-1522

23. Huang CH, Ma HM, Liau JJ, Ho FY, Cheng CK (2002) Osteolysis in failed total knee arthroplasty: a comparison of mobile-bearing and fixed-bearing knees. J Bone Joint Surg Am 84-A(12):2224-2229

24. Siddiqui O, Hung HM, O'Neill R (2009) MMRM vs. LOCF: a comprehensive comparison based on simulation study and 25 NDA datasets. J Biopharm Stat 19(2):227-246

25. Solarino G, Spinarelli A, Carrozzo M, Piazzolla A, Vicenti G, Moretti B (2014) Long-term outcome of low contact stress total knee arthroplasty with different mobile bearing designs. Joints 2(3):109-114

26. Wood A, Keenan A, Arthur C, Aitken S, Walmsley P, Brenkel I (2013) The functional outcome of total knee replacement in young patients: a 10-year matched case control study. Open J Orthopedics 3(2):128-132

\section{Submit your manuscript to a SpringerOpen ${ }^{\circ}$ journal and benefit from:}

- Convenient online submission

- Rigorous peer review

- Open access: articles freely available online

- High visibility within the field

- Retaining the copyright to your article

Submit your next manuscript at $\boldsymbol{\nabla}$ springeropen.com 